\title{
Brain processing of visual metaphors: an electrophysiological study
}

\author{
$\underline{\text { María J. Ortiz'1, M.D. Grima Murcia }{ }^{2} \text {, E. Fernandez }}$ \\ ${ }^{1}$ Dept. of Communication and Social Psychology, University of Alicante, Spain \\ ${ }^{2}$ Institute of Bioengineering, University Miguel Hernández and CIBER BBN, Spain
}

\section{Introduction}

Metaphors are pervasive in everyday language and often used in advertisements to draw attention and persuade consumers to purchase products or services. Despite it, there are many open questions regarding how metaphors are processed in the brain. In general, there are three possible models: the standard model, the parallel hypothesis, and the context-dependent hypothesis. According to the first model, metaphors take longer to process than literal statements because metaphoric interpretation should be sought only when literal interpretations are defective. The parallel hypothesis model argues that both metaphoric and literal meanings are processed concurrently and involve the same mechanisms. Finally, the context-dependent hypothesis postulates that the metaphorical meaning is directly accessed when this is made obligatory by the context.

Several researches have used Event-Related Brain Potentials (ERPs) to study the cognitive processes that underlie metaphor comprehension because they provide an online index of brain activity (Coulson, 2008). The ERP component most widely studied in metaphor processing research is the N400, which has been correlated with processing difficulty. The standard model would predict an initial N400, reflecting the literal incongruity, followed by a later ERP effect, reflecting the access of the metaphoric meaning. In contrast, the parallel hypothesis would expect N400 in literal and metaphoric sentences but with different amplitudes reflecting comprehension difficulty. 
The context-dependent hypothesis would assume that the context should modulate the N400 amplitude.

Coulson \& van Petten (2002) compare literal sentences ("He knows that whiskey is a strong intoxicant"), literal mappings ("He has used cough syrup as an intoxicant") and metaphors ("He knows that power is a strong intoxicant"). Metaphors elicit larger N400s than literal sentences, with literal mapping falling between metaphors and literals. These authors interpret such findings as being indicative of a gradient of difficulty in sentence comprehension across the three conditions consistent with the parallel model and the claim that literal and metaphoric language share some processing mechanisms but that metaphoric language requires more processing effort than literal language.

Arzouan, Goldstein \& Faust (2007a) investigate the processing differences between novel metaphors, conventional metaphors, literal sentences and meaningless expressions. Consistent with Coulson \& van Petten's conclusions mentioned above, the results show a grading in N400 amplitudes, the lowest for literal sentences, increasing slightly in negativity for conventional metaphors, then for novel metaphors and the most negative for non-semantic relation. It seems, therefore, that literal expressions are the easiest to process, whereas unrelated pairs are the most difficult are require the most effort. Other experiments replicate these results (cf. (De Grauwe, Swain, Holcomb, Ditman, \& Kuperberg, 2010; Lai, Curran, \& Menn, 2009; Schneider et al., 2014; Tartter, Gomes, Dubrovsk, \& Molholm, 2002) so this graded N400 metaphor effect seems to be further confirmed.

Although the metaphor is seen as an essential mechanism in all thought processes, most electrophysiological recording experiments have used only verbal stimuli and little is 
known about how visual metaphors are processed, in part because this field of study is more recent and complex than the study of verbal metaphor.

Researchers who have tried to enumerate the formal structures of visual metaphors in pictorial advertising distinguish three basic types. The first type involves the fusion of different objects in a hybrid image; in the second type only one object actually appears but the other is somehow suggested through the context; and in the third type at least two objects appear simultaneously. These three visual structure-types have received different names. For example, "hybrid metaphor", "contextual metaphor" and "simile" (Forceville, 2007) or "fusion", "replacement" and "juxtaposition" (Maes \& Schilperoord, 2008). The three types of visual metaphor elicit different responses (van Mulken, le Pair, \& Forceville, 2010) and can be differentiated with regard to their perceived complexity and deviation from expectation. Hybrids are considered more deviant than similes and contextual metaphors because participants might consider unlikely the fusion of two elements into a single 'gestalt'.

In this study, we measured brain electrical activity when subjects are looking at literal images and hybrid metaphors in several advertisements. We consider literal images those that show the product directly. That is, just images of the advertised objects. They are, in other words, images of the object advertised in which there is no transference of qualities between objects, as happens with visual metaphors. On the other hand, we have chosen hybrid metaphors as our stimuli because they are the most deviant and could be regarded as most similar to novel verbal metaphors. Our main aims are: (a) to determine whether visual and verbal metaphors share processing patterns, and (b) to get insights into electrophysiological processing of visual metaphors. Following procedures carried out in the most recent verbal metaphor publications, we hypothesize the 
existence of greater negative amplitude at $400 \mathrm{~ms}$ time-windows for hybrid metaphors contrasted with literal images.

\section{Material and methods}

\subsection{Participants}

Twenty-two subjects participated in the study (eleven men, eleven women; average age: 24.7; range: 19.7-33). None of the participants had any personal history of neurological or psychiatric illness, drug or alcohol abuse, or current medication, and they had normal or corrected-to-normal vision. All of them were right-handed with a laterality quotient of at least +0.4 (mean 0.8, SD: 0.2) on the Edinburgh Inventory (Oldfield, 1971). All subjects were informed about the aim and design of the study and gave their written consent for participation.

\subsection{Materials, procedure and acquisition}

Twelve pictorial advertisements collected from Internet databases were used as stimuli. Six were hybrid metaphors and six were literal images (Figure 1 and 2 show representative examples). The advertisements were chosen because they belonged to the class of monomodal metaphor, that is, the metaphor occurred in only one (visual) mode. Logos, slogans and brand names (if present) were removed from the original advertisements to avoid reading influence. In order to contextualize the image and ensure that it was understood, a text about the product advertised was included before each picture.

To ensure that there was no luminance difference across the images in the two categories, we measured the luminance of each image. An ANOVA showed no significant differences in luminance between literal and metaphoric images $(\alpha>0.05)$, 
consequently we rule out the possibility that differences in the brain processing could be due to luminance differences in the stimuli.

The subjects were comfortably seated at a distance of $0.9 \mathrm{~m}$ from the screen. Participants were informed that they were about to see a series of advertising images devoid of text, that some of the images were more complex than others, and that they had to think about what the advertiser was trying to suggest. Figure 3 summarizes the serial structure of the study. Each image was presented for 2 seconds, preceded by the contextualizing slide for 2 seconds, and followed by a black screen for 3 seconds. Then the participants were asked to indicate the answer they believed correct ( 1 or 2$)$ for each picture. The images appeared randomly and only once.

\subsection{Data acquisition}

We instructed the subjects to remain as immobile as possible, to avoid blinking during image exposure and to try to concentrate their gaze towards the centre of the monitor screen. EEG data were continuously recorded at a sampling rate of $1000 \mathrm{~Hz}$ from 64 locations using the international 10/20 system (Klem, Lüders, Jasper, \& Elger, 1999). The EEG data were recorded via cap-mounted $\mathrm{Ag}-\mathrm{AgCl}$ electrodes. A 64-channel NeuroScan SynAmps EEG amplifier (Compumedics, Charlotte, NC, USA) was used. The impedance of the recording electrodes was monitored for each subject prior to data collection and the thresholds were kept below $25 \mathrm{k} \Omega$. The subjects were seated at a distance of $0.9 \mathrm{~m}$ from the screen. All the recordings were performed in a silent room with soft lighting.

Signal processing was performed with the help of Curry 7 (Compumedics, Charlotte, NC, USA). The data were re-referenced to a Common Average Reference (CAR) 
because this was required by the statistical and analysis methods later applied. The EEG signals were filtered using a $45 \mathrm{~Hz}$ low-pass and a $1 \mathrm{~Hz}$ high-pass filters. Eye blink effects were reduced using a regression analysis in combination with artifact averaging.

EEG data in the interval $(-100,2000)$ msec from stimulus onset were analyzed in this study. Epochs with signals exceeding $\pm 75 \mu \mathrm{V}$ were excluded, since a visual inspection of the common average referenced epochs showed that signals of this magnitude were probably due to artifact. Averages for both stimulus types (literal images, metaphoric images) were computed for each person.

\subsection{Statistical analyses}

Differences in topographic patterns of activity between literal and metaphoric images were assessed using Curry 7 software (Compumedics, Charlotte, NC USA). We focused our analysis on an approach that has been widely used in psychophysiology: the examination of topographic changes in EEG activity (cf. Brunet, Murray, \& Michel, 2011; Laganaro, Valente, \& Perret, 2012; Martinovic et al., 2014; Murray, Brunet, \& Michel, 2008). This approach considers whole-scalp EEG activity elicited by a stimulus as a finite set of alternating spatially stable activation patterns, which reflect a succession of information processing stages. There are two main reasons why we used this type of analysis rather than the more traditional type based on the assessment of amplitudes and latencies of a set of predefined ERP components. First, it takes into consideration the entire time course of activity and the entire pattern of activation across the scalp by testing the global field power from all electrodes (Skrandies, 1990). Secondly, this approach is able to detect not only differences in amplitude, but also differences in underlying sources of activity. The latter is based on the fact that maps that are confirmed to be both spatially and temporally different must necessarily be the 
product of a different set of generators. However, we emphasize that the analysis of topography changes is compatible with the analysis of traditional ERP.

Topographical differences were tested through a non-parametric randomization test known as TANOVA (Topographic ANOVA), which allows investigating significant differences in global dissimilarity of EEG activity between two conditions by assessing whether the topographies are significantly different from each other on a temporal point-by-point basis. This approach was used to indicate the time windows of interest for further ERP analysis and analysis with sLORETA methodology. The significance level was $\alpha \leq 0.01$ an according with Rosenblad (2009) the required number of repetitions was chosen to be $p>1000$. Map normalization was used for the difference tests, such that the MGFP per map was equal to 1.

ERP positivity and negativity were calculated from nine sets of electrode sites (see Figure 4): anterior, central and posterior; left, midline and right. Differences in peaks were contrasted during the time-period windows indicated by the TANOVA to be significantly different.

On significant time windows, we performed sLORETA calculations for the localization of the activity in the brain (Pascual-Marqui, 2002). The Boundary Element Method (BEM) was used in the head reconstruction since it permits the localization of the source dipoles. Furthermore, BEM models are superior in non-spherical parts of the head like the temporal and frontal lobes or basal parts of the head, where spherical models exhibit systematic localizations of up to $30 \mathrm{~mm}$ (Vatta, Meneghini, Esposito, Mininel, \& Di Salle, 2010). 


\section{Results}

\subsection{Participant comprehension}

On average, participants responded correctly to the questions, with a mean of $89.4 \%$ correct (SD 12.1\%).

\subsection{EEG}

Significant differences in stimulus-elicited activity (literal versus metaphoric images) are showed in Figure $5(\mathrm{p}<0.01)$. These differences started approximately $157 \mathrm{~ms}$ after image onset an appeared in all samples. The test for differences between literal an metaphoric images yielded significant latencies in three time windows: $157-196 \mathrm{msec}$, 406-430 msec, and 580-587 msec.

\subsection{Event-related potentials (ERPs)}

ERPs were derived by averaging correctly classified trials on each condition for each participant. Figure 6 shows the ERPs generated by the two conditions (literal images and metaphoric hybrids in the frontal $(\mathrm{Fz}, \mathrm{F} 3, \mathrm{~F} 4)$, central $(\mathrm{Cz}, \mathrm{C} 3, \mathrm{C} 4)$ and parietal $(\mathrm{Pz}$, P3, P4) areas. Differences can be easily observed between the processing of hybrid metaphors and that of literal images, especially in the frontal and parietal areas. In particular, more negative waves can be seen for metaphors in earlier time windows (between 200 and $400 \mathrm{~ms}$ ) in the frontal area, while literal images produce more positive waves. In the posterior region, however, there is a significant positive wave in the metaphoric condition, at around $300 \mathrm{~ms}$. 
ANOVA was performed in the time windows of significant differences indicated by the TANOVA. We compared peak maxima or minima in order to find the location of the highest positivity in 200, 400 and $600 \mathrm{~ms}$ time windows (see Table 1).

Our results showed that in the first time interval (157-196ms), metaphoric images generated more positivity (P200) in the posterior areas. In the $406-430 \mathrm{~ms}$ time interval, literal images generated more negativity (N400) in the anterior right area, while metaphoric images did so in the posterior area. In the least significant interval (580$587 \mathrm{~ms}$ ), literal images generated positivity (P600) in the left posterior area, whereas metaphorical images did so in the right central area. Topographic maps are showed in Figure 7.

\subsection{Source analysis (sLORETA)}

A standardized Low Resolution Electromagnetic Tomography (sLORETA) analysis showed different activation patterns for the three representative time windows (figure 8). Table 2 shows the maximum current density results with sLORETA for 9579 sources. Coordinates and brain areas (Talairach coordinates) are indicated.

\section{Discussion}

The results of this study reveal two fundamental findings. Firstly, there are processing differences between literal images and hybrid metaphors, as regards both the eventrelated potentials and the current density of the cerebral activity. And secondly, parallels can be observed between the processing of hybrid metaphors and that of novel verbal metaphors. 
In the case of hybrid metaphors, there appears to be an N300-N400 pattern similar to that described by Barrett \& Rugg (1990), who in their analysis of how incongruent images are processed observed a frontal-distribution, negative-polarity component at around $300 \mathrm{~ms}$, followed by a second negative spike in the $400-\mathrm{ms}$ window. According to West \& Holcomb (2002), this N300 might indicate a specific process for the representation of objects, since this component is not observed in studies of verbalstimuli processing. These findings may mean that the semantic processing of pictorial stimuli takes place within a neuronal system separate from that involved in the processing of words and utterances. In our study, however, the N300 value only occurs with hybrid metaphors and is absent with literal images, therefore it cannot be considered to reflect specific pictorial-stimuli processing. In this framework, we believe it is much more likely to be the same pattern described by Barrett \& Rugg (1990) in response to incongruent images. In other words: the N300-N400 pattern occurs with hybrid metaphors and not with literal images because the former are incongruent and the latter are not.

On the other hand, the results of the analysis of brain topographies and source distributions also show that there are specific differences in the processing of literal and metaphorical images, especially at early stages. Thus, greater frontal activity is observed with metaphors at a time window of $157-196 \mathrm{~ms}$, while later, in the $406-430 \mathrm{~ms}$ time window, there is bilateral activity in the frontal lobes and the right temporal region. Furthermore, in the time window of 580-587 ms, metaphorical images elicit noticeable activity only in the right temporal area. By contrast, literal images induce, in general, less activity. 
These results support the hypothesis that literal images and hybrid metaphors are processed differently. While literal images tend to stimulate general activity in both hemispheres leading to activity exclusively located in the left hemisphere in the final time-window, greater relative activity is observed in the right hemisphere in the case of hybrid metaphors. These finding coincide with those of Arzouan, Goldstein, \& Faust (2007b), Pobric, Mashal, Faust, \& Lavidor, (2008), Schimdt \& Seger (2009) and Lai et al (2015). In the first of these, novel verbal metaphors produced asymmetric activityvolume and current-density between the hemispheres, with greater relative activity in the right hemisphere in comparison with effects produced by literal utterances. The study carried out by Pobric, Mashal, Faust, \& Lavidor (2008) using transcranial magnetic simulation suggests that the right hemisphere might play a vital role in the comprehension of novel metaphors. Schimdt \& Seger (2009) and Lai et al (2015) with fMRI conclude that the relative contribution of the right hemisphere was greater for metaphors.

The specific areas of maximum activity are, in the case of metaphors, the Medial Frontal Gyrus (at a time window of $200 \mathrm{~ms}$ ), the Gyrus Rectus (at a time window of 400 $\mathrm{ms}$ ) and the Right Superior Temporal Gyrus (at a time window of $600 \mathrm{~ms}$ ). These results coincide with those of Arzouan, Goldstein \& Faust (2007b) who found that novel metaphors produced greater activity in the Superior Frontal Gyri within the 350$450 \mathrm{~ms}$ time-window. Though their time-window was not exactly the same as ours (406-430 ms), the localization of this greater activity is very similar, since the Gyrus Rectus and the Superior Frontal Gyri are neighbouring areas. 


\section{Conclusions}

The first conclusion to be drawn from this study is that there are striking differences in the way literal and metaphoric advertising images are processed. Metaphoric images induce more negative amplitudes than literal ones do in early time-windows, as well as greater frontal and bilateral activity. The data may be interpreted to suggest that hybrid metaphors, in contrast to literal images, initially appear to be incongruent, requiring more activation in order to process them.

One of the main aims of our study was to determine whether visual metaphors follow the same processing-pattern as that already described for verbal metaphors. Our data show coincidences between hybrid metaphors and novel verbal metaphors in the N400 effect, in which greater relative activity is detected in the right hemisphere than in the left, with maximum activity being localized in closely-linked areas (the gyrus rectus and the superior frontal gyri).

The results of our research open up possibilities for further studies, since they support the idea that metaphor, either verbal or visual, might be processed by activating a common neuronal substrate. More data are required to confirm this hypothesis; it will be necessary, for instance, to plan research using both visual and verbal metaphors based on a common metaphorical concept or presenting two types of fused objects, nonsensical and metaphorical. Furthermore, future studies should determine whether processing differences exist between the three types of visual metaphor described here (hybrid, contextual and simile), or whether such differences are only apparent. Deeper, more complete knowledge of the processing of visual metaphors is required, in order to understand more precisely the workings of the brain and also to determine to what 
extent metaphor is not simply a verbal device but also has a cognitive origin as Conceptual Metaphor Theory suggests.

\section{Acknowledgements:}

This research was carried out thanks to Alicante University's emerging research grant system (GRE-26), the Bidons Egara Research Chair of the University Miguel Hernández and grant MAT2015-69967-C3-1-R. We thank Jose A. Moya and Bryn Moody for their support and valuable comments.

\section{References}

Arzouan, Y., Goldstein, A., \& Faust, M. (2007a). Brainwaves are stethoscopes: ERP correlates of novel metaphor comprehension. Brain Research, (1160), 69-81.

Arzouan, Y., Goldstein, A., \& Faust, M. (2007b). Dynamics of hemispheric activity during metaphor comprehension: Electrophysiological measures. NeuroImage, 36(1), 222-231. doi:S1053-8119(07)00126-7 [pii]

Barrett S.E. \& Rugg M.D. (1990). Event-related potentials and the semantic matching of pictures. Brain and Cognition, 14(2), 201-212.

Brunet, D., Murray, M. M., \& Michel, C. M. (2011). Spatiotemporal analysis of multichannel EEG: CARTOOL. Computational Intelligence and Neuroscience, 1, $1-15$.

Coulson, S. (2008). Metaphor comprehension and the brain. In R.W. Gibbs (Ed.) Metaphor and Thought, 3rd edition. Cambridge \& New York: Cambridge University Press.

Coulson, S., Van Petten, C. (2002). Conceptual integration and metaphor: An eventrelated potential study. Memory and Cognition, 958-968. 
De Grauwe, S., Swain, A., Holcomb, P. J., Ditman, T., \& Kuperberg, G. R. (2010). Electrophysiological insights into the processing of nominal metaphors. Neuropsychologia, 48(7), 1965-1984. doi:10.1016/j.neuropsychologia.2010.03.017

Forceville, C. (1996). Pictorial metaphor in advertising. New York: Routledge.

Forceville, C. (2007). Multimodal metaphor in ten dutch TV commercials. Public Journal of Semiotics, 1, 19-51.

Klem, G. H., Lüders, H. O., Jasper, H. H., \& Elger, C. (1999). The ten-twenty electrode system of the international federation. . Electroencephalography and Clinical Neurophysiology Supplement, 52, 3-6.

Laganaro, M., Valente, A., \& Perret, C. (2012). Time course of word production in fast and slow speakers: A high density ERP topographic study. NeuroImage, 59(4), $3881-3888$.

Lai, V. T., Curran, T., \& Menn, L. (2009). Comprehending conventional and novel metaphors: An ERP study. Brain Research, 1284(0), 145-155. doi:http://dx.doi.org/10.1016/j.brainres.2009.05.088

Lai, V. T., van Dam, W., Conant, L. L., Binder, J. R., \& Desai, R. H. (2015). Familiarity differentially affects right hemisphere contributions to processing metaphors and literals. Frontiers in Human Neuroscience, 9 (44). http://doi.org/10.3389/fnhum.2015.00044

Maes, A. \& Schilperoord J. (2008). Classifying visual rhetoric. conceptual and structural heuristics in interpreting and classifying visual rhetoric. In B. J. Phillips, 
\& E. F. McQuarrie (Eds.), Go figure: New directions in advertising rhetoric. New York: Sharpe.

Martinovic, J., Jones, A., Christiansen, P., Rose, A. K., Hogarth, L., \& Field, M. (2014). Electrophysiological responses to alcohol cues are not associated with pavlovianto-instrumental transfer in social drinkers. PLoS ONE, 14(9)

Murray, M. M., Brunet, D., \& Michel, C. M. (2008). Topographic ERP analyses: A step-by-step tutorial review. Brain Topography, 20(4), 249-264.

Oldfield, RC. (1971). The assessment and analysis of handedness: the Edinburgh inventory, Neuropsychologia, 9 (1), 97-113.

Pascual-Marqui, R. D. (2002). Standardized low-resolution brain electromagnetic tomography (sLORETA): Technical details. Methods and Findings in Experimental and Clinical Pharmacology, 24, 5-12.

Pobric, G., Mashal, N., Faust, M., \& Lavidor, M. (2008). The role of the right cerebral hemisphere in processing novel metaphoric expressions: A transcranial magnetic stimulation study. Journal of Cognitive Neuroscience, 20(1), 170-181.

Rosenblad, A. (2009). B. F. J. manly: Randomization, bootstrap and monte carlo methods in biology, third edition. Computational Statistics, 24(2), 371-372. doi:10.1007/s00180-009-0150-3

Schmidt, G.L. \& Seger, C.A. (2009). Neural correlates of metaphor processing: The roles of figurativeness, familiarity and difficulty. Brain and Cognition, 71, 3, 375386, dx.doi.org/10.1016/j.bandc.2009.06.001. 
Schneider, S., Rapp, A. M., Haeussinger, F. B., Ernst, L. H., Hamm, F., Fallgatter, A. J., \& Ehlis, A. C. (2014). Beyond the N400: Complementary access to early neural correlates of novel metaphor comprehension using combined electrophysiological and haemodynamic measurements. Cortex. 53, 45-59. doi:10.1016/j.cortex.2014.01.008

Skrandies, W. (1990). Global field power and topographic similarity. Brain Topography, 3(1), 137-141.

Tartter, V., Gomes, H., Dubrovsk, B., \& Molholm, S., R. (2002). Novel metaphors appear anomalous at least momentarily: Evidence from N400. Brain and Language, 80, 488-509.

Vatta, F., Meneghini, F., Esposito, F., Mininel, S., \& Di Salle, F. (2010). Realistic and spherical head modeling for EEG forward problem solution: A comparative cortexbased analysis. Computational Intelligence and Neuroscience, 1-11.

van Mulken, M., le Pair, R., \& Forceville, C. (2010). The impact of perceived complexity, deviation and comprehension on the appreciation of visual metaphor in advertising across three european countries. Journal of Pragmatics, 42 (12), 34183430

West, W. C. \& Holcomb, P. J. (2002). Event-related potentials during discourse-level semantic integration of complex pictures. Cognitive Brain Research, 13(3), 363375. 


\section{Figure captions}

Figure 1. Literal images.

Figure 2. Hybrid metaphors.

Figure 3. Experimental design. The sequence of stimuli was presented in continuous mode by using commercial stimulus presentation software (STIM2, Compumedics, Charlotte, NC, USA).

Figure 4. Electrode sites of interest: anterior, central and posterior on the left, midline and the right side of the head. The two active electrodes that are shown are REF (reference) which acts as a recording reference and GND which serves as a ground.

Figure 5. Time points of significant differences in EEG activity for the 2 contrasts (literal versus metaphor), as indicated by the TANOVA analysis, depicting 1 minus pvalue across time. Significant $p$ values are plotted $(\alpha \leq 0.01)$. The three vertical rectangles contain intervals with significant differences.

Figure 6. Grand average ERP waveforms elicited by literal images (blue) and hybrid metaphors (green) from frontal scalps (Fz, F3, F4), central scalps (Cz, C3, C4) and parietal scalps (Pz, P3, P4). Negative voltage is plotted downwards.

Figure 7. Topographic maps at significant intervals.

Figure 8. Activation maps for sLORETA.

Table 1. Results of ANOVA averages (metaphoric versus literal comparison).

Table 2. Compared Cartesian areas between literal images and metaphors. 